\title{
PAPERS
}

\section{Diffuse oesophageal spasm: diagnosis by ambulatory 24 hour manometry}

\author{
C P Barham, D C Gotley, A Fowler, A Mills, D Alderson
}

\begin{abstract}
Background-Diffuse oesophageal spasm (DOS) is a potential cause of intermittent chest pain and/or dysphagia. In the past, the diagnosis of DOS has relied on criteria obtained from standard oesophageal manometry (more than one simultaneous contraction in a series of 10 wet swallows with the rest being peristaltic). As symptoms are intermittent, however, 24 hour manometry may well be more suited to its investigation.

Aims-To determine the ability of 24 hour manometry to detect the symptomatic contractions of DOS and to compare standard, laboratory based manometry with 24 hour manometry in its diagnosis. Patients-Three hundred and ninety consecutive patients referred with suspected oesophageal disorders.

Methods-Standard laboratory based manometry and 24 hour outpatient manometry.

Results-Sixteen patients were classified by 24 hour manometry as having DOS on the basis of painful contractions (spasms) of excessive duration and increased amplitude. Laboratory based manometry failed to detect the majority of these patients with DOS (14/16), and 53/55 were incorrectly labelled as having DOS on the basis of asymptomatic manometric findings. Conclusion-The detection of symptomatic DOS requires 24 hour manometry. (Gut 1997; 41: 151-155)
\end{abstract}

Keywords: oesophagus; motility disorders; ambulatory manometry; diffuse oesophageal spasm; non-cardiac chest pain

University

Department of

Surgery, Bristol Royal

Infirmary, UK

C P Barham

D C Gotley

A Fowler

A Mills

D Alderson

Correspondence to:

Mr C P Barham, University

Department of Surgery,

Bristol Royal Infirmary,

Bristol BS2 8HW, UK.

Accepted for publication 10 March 1997

Diffuse oesophageal spasm (DOS) is a clinical syndrome characterised by symptoms of retrosternal chest pain and/or intermittent dysphagia. The chest pain can be severe and is frequently mistaken as cardiac in origin. Initially described by Osgood in $1889,{ }^{1}$ the first manometric descriptions were not until $1958^{2}$ and $1964 .{ }^{3}$ Before manometry, the diagnosis relied on the symptom complex and the radiological findings ${ }^{4}$ of a normal oesophageal lumen, failure of peristaltic propagation, and simultaneous oesophageal contractions, sometimes resulting in a beaded appearance. A variety of radiological appearances and terminologies have been used such as "pseudodiverticulosis", "segmental spasms", and "corkscrew oesophagus", although in most cases the diagnosis would be missed by barium swallow as the oesophagus usually appears normal.

Early manometric studies described the salient features as the presence of simultaneous lower oesophageal contractions in response to swallows, occasional very high amplitude contractions, and contractions with prolonged duration and multiple peaks. Contractions that were prolonged were most likely to correspond to patient symptoms. ${ }^{5}$ With refinements in equipment and methodology (water perfused and solid state systems, and the standard use of the 10 wet swallow test), some of the primary features of DOS were reduced in importance while the simultaneous nature of contractions became the main diagnostic criterion. Studies in control subjects and symptomatic patients have led to a manometric definition of DOS as "two or more simultaneous contractions interspersed with normal peristalsis in a series of ten wet swallows". ${ }^{6}$ Contraction amplitudes and durations are not now required to be abnormal to make the diagnosis, and it is not considered necessary to correlate symptoms with manometric findings. ${ }^{7}$

As DOS produces intermittent symptoms ${ }^{8} 9$ 24 hour manometry (due to its ability to correlate symptoms with manometric abnormalities) may well be more suited to its investigation. The aim of the study, therefore, was to examine patients with suspected DOS by 24 hour combined $\mathrm{pH} /$ manometry and compare the findings with standard laboratory based oesophageal manometry.

\section{Materials and Methods}

Between April 1990 and December 1994, 390 patients with symptoms thought to arise from the oesophagus were referred to the oesophageal motility laboratory of the University Department of Surgery, Bristol Royal Infirmary. The recording system and automated analysis software were developed in collaboration with Gaeltec Ltd, Dunvegan, Scotland. The two connected recording catheters comprised an antimony $\mathrm{pH}$ probe (Synectics, Sweden) positioned to lie $5 \mathrm{~cm}$ above the manometrically determined lower oesophageal sphincter (LOS), and a five channel pressure 
TABLE 1 Minimum criteria for the diagnosis of diffuse oesophageal spasm based on a 24 hour manometry study

(1) Duration of "spasm" contraction greater than 15 seconds

(2) Maximum amplitude of "spasm" contractions greater than $200 \mathrm{~mm} \mathrm{Hg}$

(3) "Spasm" contractions should involve greater than $10 \mathrm{~cm}$ of oesophageal body

(4) Symptoms (which should occur with some of the contractions) of chest pain and/or dysphagia should occur during or within 30 seconds of the contraction ending

TABle 2 Patient details

\begin{tabular}{|c|c|c|c|c|c|c|c|c|}
\hline & Patient & Age & Sex & $P M H$ & Presentation & Swallow & Endoscopy & $\begin{array}{l}\text { Percentage } \\
\text { time } p H<4\end{array}$ \\
\hline 1 & $\mathrm{JN}$ & 48 & $M$ & $\mathrm{DU}$ & $\mathrm{CP}$ & Normal & Normal & 4.0 \\
\hline 2 & PM & 53 & $\mathrm{~F}$ & Nil & $\mathrm{CP}$ & Normal & - & 1.4 \\
\hline 3 & $\mathrm{KJ}$ & 25 & $\mathrm{~F}$ & Nil & $\mathrm{CP}$ & Normal & Normal & 0.8 \\
\hline 4 & MW & 63 & $\mathrm{~F}$ & $\mathrm{Dp}$ & $\mathrm{D}+\mathrm{CP}$ & TC & SC & 5.7 \\
\hline 5 & $\mathrm{CM}$ & 35 & $\mathrm{~F}$ & Nil & $\mathrm{D}+\mathrm{CP}$ & Normal & - & 0.2 \\
\hline 6 & JB & 36 & M & Nil & $\mathrm{D}+\mathrm{CP}$ & TC & Normal & 0.1 \\
\hline 7 & RC & 48 & M & Nil & $\mathrm{D}+\mathrm{CP}$ & TC & Normal & 0.4 \\
\hline 8 & VJ & 72 & $\mathrm{~F}$ & Nil & $\mathrm{D}+\mathrm{CP}$ & Corkscrew & Normal & 1.6 \\
\hline 9 & MH & 57 & $\mathrm{~F}$ & Nil & $\mathrm{D}+\mathrm{CP}$ & Normal & Normal & 0.9 \\
\hline 10 & $\mathrm{ZF}$ & 75 & $\mathrm{~F}$ & Nil & $\mathrm{D}$ & Normal & Diverticulum & 0.9 \\
\hline 11 & DB & 67 & M & Nil & $\mathrm{CP}$ & Normal & Normal & 3.0 \\
\hline 12 & DB & 45 & M & Nil & $\mathrm{D}+\mathrm{CP}$ & - & Normal & 3.4 \\
\hline 13 & GB & 34 & M & Nil & $\mathrm{D}+\mathrm{CP}$ & Normal & Normal & 5.2 \\
\hline 14 & $\mathrm{BH}$ & 64 & $\mathrm{~F}$ & $\mathrm{Dp}$ & $\mathrm{D}+\mathrm{CP}$ & Corkscrew & Normal & 0.1 \\
\hline 15 & $\mathrm{DE}$ & 61 & $\mathrm{~F}$ & Nil & $\mathrm{D}+\mathrm{CP}$ & Normal & Normal & 0.1 \\
\hline 16 & $\mathrm{LM}$ & 38 & $\mathrm{~F}$ & Nil & $\mathrm{D}+\mathrm{CP}$ & Normal & Normal & 0 \\
\hline
\end{tabular}

DU, duodenal ulceration; Dp, depression; CP, chest pain; D, dysphagia; TC, tertiary contractions; SC, segmental contractions; PMH, past medical history.

catheter. The solid state manometry catheter (CTO-5/Sphinctometer, Gaeltec Ltd) contained three oesophageal body pressure transducers (the lowest at the same level as the $\mathrm{pH}$ probe), a $6 \mathrm{~cm}$ long sphincter monitoring device, and a gastric pressure transducer, all spaced $5 \mathrm{~cm}$ apart. ${ }^{10}$ Both catheters were connected to a 24 hour recording unit (7-MPR, Gaeltec Ltd) which contained a microprocessor for data compression and allowed connection to a computer for subsequent data analysis.

For all 390 patients, the manometric study involved an initial laboratory based investiga-

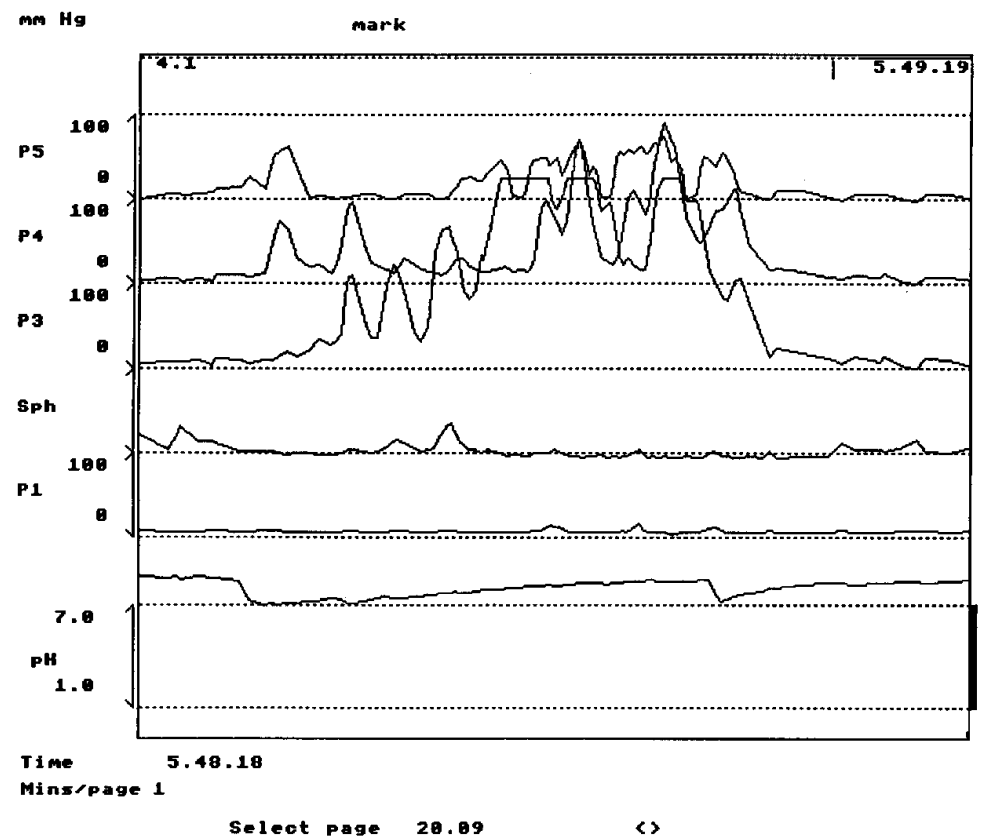

Figure 1: Typical spasm contraction (amplitude $>200 \mathrm{~mm} \mathrm{Hg}$, duration $>15$ seconds, multi-peaked) occurring in the oesophageal body and causing pain (note the event marks at the top). The start of the contractions appears peristaltic but due to their duration the majority of the contraction sequence is simultaneous. P3-P5, oesophageal transducers; Sph, sphinctometer; P1, gastric transducer; $p H$, oesophageal $p H$ probe. tion to determine the mean LOS pressure by station pull-through and then 10 wet swallows of $5 \mathrm{ml}$ water at 30 second intervals. At the completion of this standard study, the two catheters were secured in position to the nose by adhesive tape and a 24 hour ambulatory outpatient recording was commenced. Patients were instructed to behave and eat as they would on a typical day but the importance of completing a diary sheet and marking symptomatic events with a button on the recording unit was stressed. At the completion of the 24 hour study, the data were downloaded onto the computer for analysis.

Data were analysed to determine if the patients' symptoms were due to acid reflux or an oesophageal motor abnormality. The diagnosis of DOS by station pull-through manometry was made by the presence of two or more simultaneous contractions, interspersed with normal peristalsis, in a series of 10 wet swallows (conventional definition). There are at present no widely accepted manometric criteria for the diagnosis of DOS based on 24 hour manometry though some attempts have been made. ${ }^{11}$ We believed that DOS should be characterised by the presence of contractions of excessive amplitude and prolonged duration with multiple peaks (fig 1 and table 1 ). We have found that only this type of contraction unequivocally produces chest pain. In addition this type of contraction does not occur in control subjects or in patients with reflux disease alone. Furthermore, we felt that a temporal relationship should exist between these types of contractions and symptoms of chest pain and/or dysphagia as other groups have also identified these contractions in patients with non-cardiac chest pain. ${ }^{11}{ }^{12}$ Using this definition, to be temporally related to a "spasm" contraction, the pain (or dysphagia) had to occur during or within 30 seconds of the contraction finishing. This definition was applied retrospectively to the patients studied. The manometric diagnosis from the conventional laboratory based study was compared with the diagnosis from the 24 hour study.

\section{Results}

Of the 390 patients studied, the 24 hour $\mathrm{pH} /$ manometry test revealed that 177 had gastro-oesophageal reflux (time $\mathrm{pH}<4$ greater than $4 \%$ and typical symptoms correlated to oesophageal acidification), 30 had achalasia, and 52 had non-specific motor abnormalities not correlated with symptoms; in 115 no abnormality could be demonstrated. Sixteen were found to have DOS as defined above. Of these 16 patients, six were male and 10 female with a median age of 48 years (range 25-75). All 16 patients, complained of either chest pain alone $(n=4)$, dysphagia alone $(n=1)$, or both chest pain and dysphagia $(n=11)$. Fourteen underwent endoscopy and 15 a barium swallow (13 underwent both procedures) prior to the 24 hour manometry study (table 2). Following the barium swallow two patients were reported to have a corkscrew oesophagus; in three patients tertiary contractions were observed, and 10 were reported as normal. At 


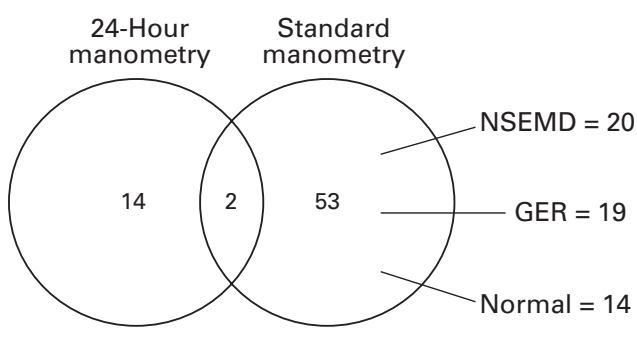

Figure 2: The diagnosis of DOS by 24 hour manometry and standard laboratory based manometry.

endoscopy one patient was noted to have a distal oesophageal diverticulum, segmental contractions were reported in another, and in 12 no abnormality was detected. In none of the 16 patients was there endoscopic or radiological evidence of reflux disease. Only $2 / 16$ patients had medical conditions requiring medication known to affect smooth muscle (depression treated with doxepin in one and fluoxetine in the other) and none had undergone surgery to the upper gastrointestinal tract.

Fifty five subjects had two or more simultaneous contractions during the standard manometry study. During the 24 hour study, however, only two of these patients $(3.6 \%)$ had symptomatic spasm contractions (DOS). Twenty of these 55 had other non-specific motor abnormalities (low amplitude contractions, poor peristaltic propagation where contractions appearing in the upper oesophagus failed to reach the distal end, or an increased frequency of low amplitude but normal duration simultaneous contractions). There was no correlation between any of these events and symptoms. Nineteen patients had symptomatic reflux with poor motility (symptoms related to reflux, not to motor disorder) and in 14 no abnormality could be demonstrated (fig 2).

Sixteen patients had symptomatic spasm contractions during the 24 hour study. Five of these patients complained of dysphagia to solids during eating and in these patients

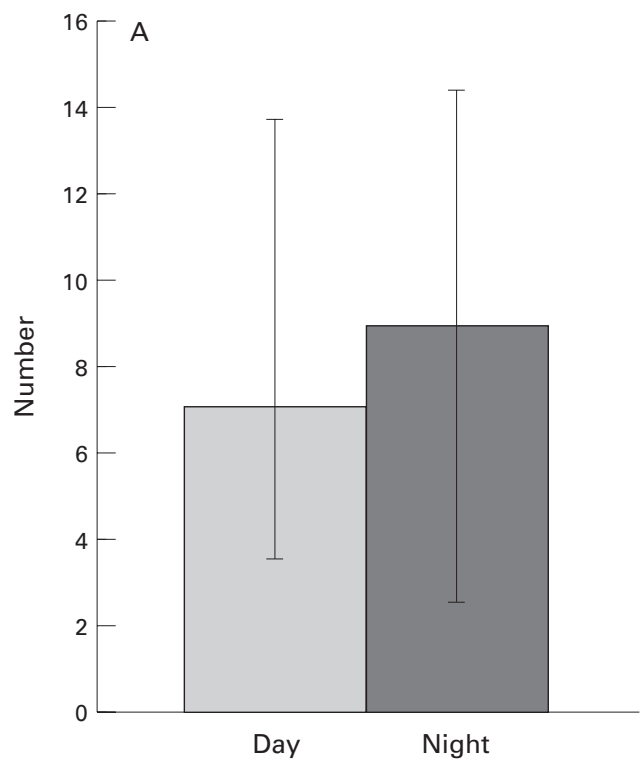

abnormal spasm contractions accounted for all episodes of dysphagia. The remaining patients had normal peristaltic contractions during their meals. Fourteen of these 16 patients had a normal acid reflux study $(\mathrm{pH}<4$ for less than $4 \%$ of the recording). In two the reflux time was only just abnormal $(5.2 \%$ and $5.7 \%$, respectively). As a group the median percentage $\mathrm{pH}$ time was 0.9 (interquartile (IQ) range $0.1-3.0)$. In no patient was acid reflux responsible for a chest pain episode and no spasm contraction occurred during an acid reflux episode.

All symptomatic spasm waves had durations of greater than 15 seconds, amplitudes of greater than $200 \mathrm{~mm} \mathrm{Hg}$, and multiple peaks (fig 1 and fig 4b). In 14 patients symptomatic contractions occurred both during the day and at night. One patient failed to get spasm contractions during the night and in a further patient no nocturnal recording was obtained due to equipment malfunction. Nocturnal contractions characteristically started about two to three hours after commencing sleep and often woke the patient from sleep (seven patients). The median number of night time spasm sequences was nine (IQ range 2.5-14.5) with a median frequency of 1.2 per hour (IQ range $0.4-2.0$ per hour). The median number of day time spasms was seven (IQ range 3.5-13.8) with a frequency of 0.6 per hour (IQ range $0.3-1.1$ per hour) (fig 3). Fourteen of the 16 patients with abnormal symptomatic "spasm" contractions during the 24 hour study had a normal standard laboratory manometry study (fig 4). Two of these 14 patients did, however, have contractions of long duration, excessive amplitude, and multiple peaks (spasms) at some time during the laboratory study (noted either before, after, or in the interval between their 10 standard wet swallows).

Not all spasm contractions were symptomatic and not all symptomatic episodes marked on the diary sheet were temporally

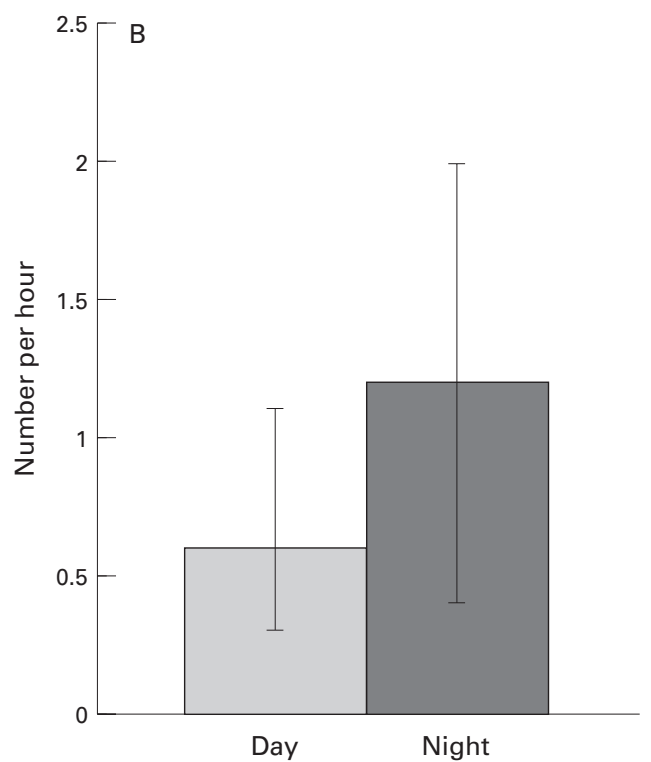

Figure 3: The number (A) and frequency (B) of spasm contractions occurring during the day and at night. Results expressed as medians and interquartile ranges. Differences were not significant. 
related to a spasm contraction. The median number of spasm contractions that produced symptoms over the 24 hours was 2.5 (IQ range $1-4)$. The median percentage of spasm contractions producing symptoms over 24 hours was 9.2 (IQ range 3.2-19.1). Spasm contractions at night were not felt unless they woke the patient from sleep (seven patients were woken by chest pain). The median percentage of symptomatic episodes recorded on the diary

A

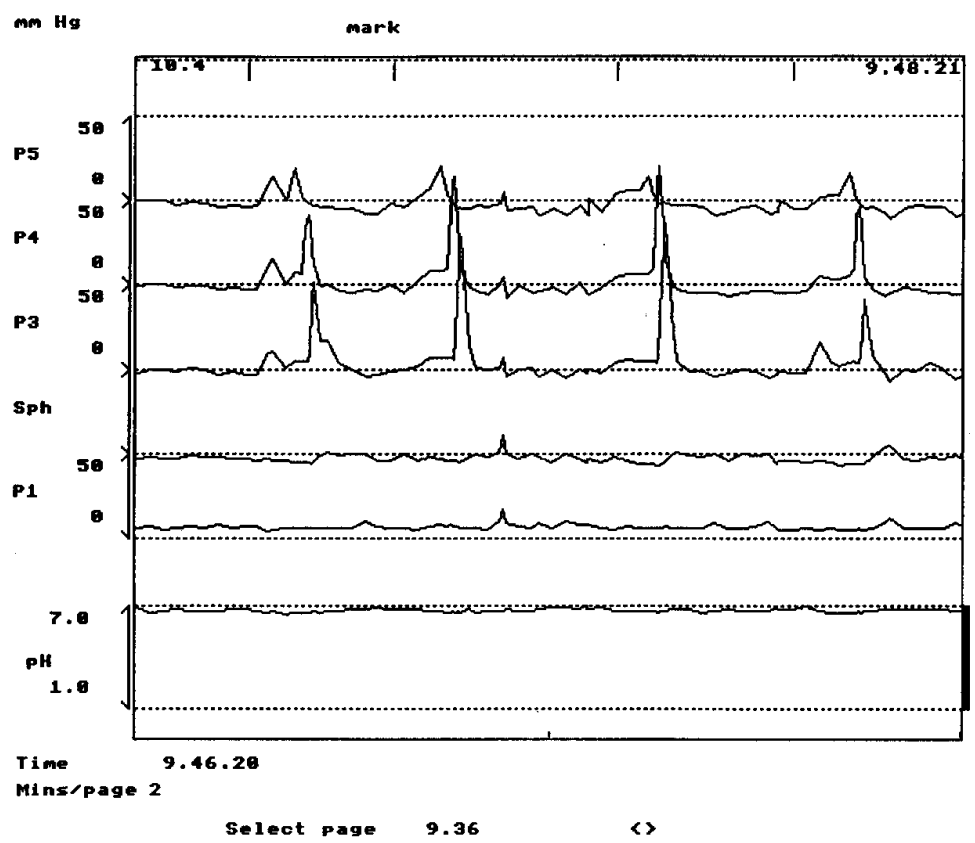

B

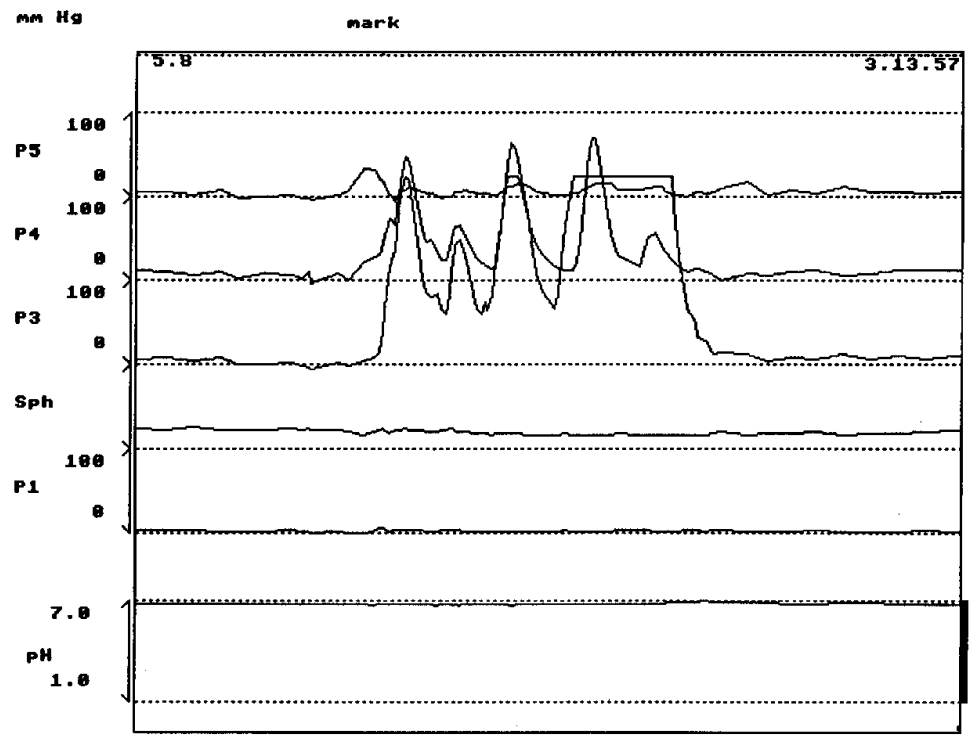

Time 3.12 .56

Mins/page 1

Select page $\mathbf{3}$.8B 《3

Figure 4: (A) Completely normal standard laboratory based manometry study. Note normal peristalsis to marked wet swallows. Contraction amplitudes are $<100 \mathrm{~mm} \mathrm{Hg}$. (B) Typical spasm contractions of diffuse oesophageal spasm recorded during the ambulatory study of the same patient. Pressure scale in (B) is $0-100 \mathrm{~mm} \mathrm{Hg}$ per channel so peak contraction amplitude of the spasm contraction is $>230 \mathrm{~mm} \mathrm{Hg}$. sheet that correlated with oesophageal spasms was 73.4 (IQ range 27.3-100).

\section{Discussion}

The question of whether the oesophagus is a cause of chest pain has vexed investigators for many years. The good correlation between oesophageal acidification (demonstrated by 24 hour $\mathrm{pH}$ studies) and symptoms, and the subsequent alleviation of symptoms by therapies which prevent oesophageal acidification makes it easy to understand and accept the oesophagus as the source of pain in reflux disease. For motility disorders, with the exception of achalasia, the link between symptoms and abnormalities in a manometry study are not so readily established. Unfortunately there has been a tendency to accept the oesophagus as a cause of chest pain on the basis of minor manometric variations ${ }^{13}{ }^{14}$ without a correlation to symptoms or treatment. This must inevitably overestimate the true role of the oesophagus as a source of pain. At the same time, a test which ignores symptoms may underestimate genuine oesophageal abnormalities, particularly when symptoms are intermittent and unlikely to occur during a short term test.

A variety of labels have been attached to certain manometric findings with "nutcracker oesophagus" and "diffuse oesophageal spasm" being the two conditions thought most likely to cause or be associated with chest pain. The present definition of diffuse oesophageal spasm is largely the result of work carried out by Richter et al who first defined normal oesophageal motility in a large group of controls ${ }^{15}$ and then used these data to define achalasia, nutcracker oesophagus, and DOS. ${ }^{6}$ As normal subjects produced nine or 10 peristaltic contractions to wet swallows, those who demonstrated fewer than nine peristaltic contractions with the other contractions being simultaneous were grouped separately. As this group of patients had been referred with symptoms of chest pain (and as previous studies of DOS had shown a high incidence of simultaneous contractions), this manometric abnormality became the definition of diffuse oesophageal spasm. So accepted is this definition, that strong contractions are not now thought to be important at all. ${ }^{7}$ In contrast, strong peristaltic contractions are thought to be associated (though not temporally) with chest pain in nutcracker oesophagus which is distinguished from DOS not by type, severity, or site of pain but rather by a manometric finding of 10 high amplitude peristaltic wet swallows. ${ }^{16}$ Other abnormalities such as non-propagated contractions, low amplitude contractions (more than two standard deviations below the normal range), or occasional absent contractions have been termed non-specific motor abnormalities.

In a condition that produces intermittent symptoms, any manometric or radiological investigation which demonstrates abnormalities when no symptoms are occurring can be, at best, only a marker for what might occur during symptoms. In an attempt to induce typical chest pain and correlate this with 
manometric abnormalities the cholinergic agent edrophonium has been used by many investigators. A negative response however, does not exclude DOS and typical manometric features may be induced without symptoms. ${ }^{17}$ Consequently its use as a diagnostic test is also limited. With the advent of 24 hour manometry, it has become possible to correlate symptoms with oesophageal motor abnormalities. Using a combined $\mathrm{pH}$ and manometric recording system, the present study has confirmed the findings of others that the most common diagnosis in patients referred to a motility laboratory with chest pain is acid reflux. ${ }^{9} 1819$

Some oesophageal contractions however do appear to cause pain and these contractions have prolonged durations and excessive amplitudes, ${ }^{11} 12$ often with multiple peaks. It is only this type of contraction that we have found unequivocally to produce chest pain. In addition this type of contraction does not occur in control subjects or in patients with reflux disease alone. Multiple peaks alone, however, do not cause pain as multi-peak low amplitude contractions with short durations were asymptomatic. Simultaneous contractions are common during 24 hour manometric studies in control subjects and in patients with manometric abnormalities ${ }^{20}$; they cannot by themselves, therefore, be used as the 24 hour manometric definition of DOS. In addition, simultaneous contractions of normal amplitude and duration do not cause pain. The number of spasm contractions and the number of symptomatic spasm contractions varied between patients. This finding probably reflects the differences in disease severity of the patient population and the day to day variations of individual patients in a condition that produces intermittent symptoms.

A further feature of patients with spasm contractions is the nocturnal occurrence of the spasms which, if severe, can cause arousal from sleep. The explanation for this is not clear as most long term manometry studies have shown a reduction in oesophageal contraction frequency during the night. When oesophageal spasm contractions occur during eating they may also cause dysphagia, as the whole oesophagus contracting at the same time, for long durations, cannot transport food or liquid to the stomach. While only five of the patients experienced dysphagia during the ambulatory study this was one of the main presenting complaints prompting medical referral in 12 of the 16 patients. Only two of the 16 patients had simultaneous "spasm" contractions in response to some of the wet swallows during the conventional motility study while a further two had abnormal contractions at some time during their laboratory study. At best, therefore, only four of the 16 patients would have been diagnosed as having DOS on the basis of the conventional motility study. The remainder of the patients with these spasm contractions had completely normal standard motility investigations (most also had normal barium swallows and endoscopies) and would have remained undiagnosed by conventional investi- gations. By contrast 55 patients of the 390 referred to this laboratory demonstrated two or more simultaneous contractions interspersed with normal oesophageal peristalsis during their standard manometric investigation. Only two of these had symptomatic spasm contractions during their 24 hour study; the rest had generally poor motility (frequent low amplitude simultaneous contractions, frequent nonpropagated contractions) without any symptoms or poor motility with symptomatic reflux.

It is proposed that new diagnostic criteria based on 24 hour manometry are needed for the classification of oesophageal motor abnormalities so that patients who do have DOS are not missed and those with minor manometric variations are not erroneously labelled with an abnormality and hence treated inappropriately. The standard laboratory based manometric definition of diffuse oesophageal spasm should no longer be used. An acceptable definition for DOS would be the presence of multi-peaked contractions over at least $10 \mathrm{~cm}$ of the oesophageal body lasting longer than 15 seconds and with maximum amplitudes of greater than $200 \mathrm{~mm} \mathrm{Hg}$. Some of these spasm contractions should produce symptoms of chest pain and/or dysphagia. At other times normal peristaltic contractions should be present.

1 Osgood H. A peculiar form of oesophagismus. Boston Medical Sciences fournal 1889; 120: 401-5.

2 Creamer B, Donoghue FE, Code CF. Pattern of esophageal motility in diffuse spasm. Gastroenterology 1958; 34: 782-96.

3 Roth HP, Fleshler B. Diffuse esophageal spasm. Ann Intern Med 1964; 61: 914-23.

4 Schmidt HW. Diffuse spasm of the lower half of the esophagus. Am F Dig Dis 1939; 6: 693

5 Fleshler B. Diffuse esophageal spasm. Gastroenterology 1967; 52: 559-64.

6 Castell DO, Richter JE, Dalton CB (eds). Esophageal motility testing. New York: Elsevier Science, 1987.

7 Dalton CB, Castell DO, Henson EG, Wu WC, Richter JE. Diffuse esophageal spasm: a rare motility disorder not characterised by high amplitude contractions. Dig Dis Sci 1991; 36: 1025-8.

8 Browning TH. Diagnosis of chest pain of esophageal origin. Dig Dis Sci 1990; 35: 289-93.

9 Hewsen EG, Dalton CB, Richter JE. Comparison of esophageal manometry, provocative testing and ambulatory monitoring in patients with unexplained chest pain. Dig Dis Sci 1990; 35: 302-9.

10 Barham CP, Gotley DC, Miller R, Mills A, Alderson D. Pressure events surrounding acid reflux episodes and acid clearance in ambulant healthy control subjects. Gut 1993; 34: 444-9.

11 Stein HJ, DeMeester TR, Eypasch EP, Klingman RR. Ambulatory 24-hour esophageal manometry in the evaluation of esophageal motor disorders and noncardiac chest pain. Surgery 1991; 110: 753-63.

12 Janssens J, Vantrappen G, Ghillebert G. 24-Hour recording of esophageal pressure and $\mathrm{pH}$ in patients with noncardiac chest pain. Gastroenterology 1986; 90: 1978-84.

13 Cohen S. Noncardiac chest pain. The crumbling of the sphinx. Dig Dis Sci 1989; 34: 1649-50.

14 Valori RM. Nutcracker, neurosis or sampling bias. Gut 1990; 31: 736-7.

15 Richter JE, Wu WC, Johns DN, Blackwell JN, Nelson JL, Castell JA, et al. Esophageal manometry in 95 healthy adult volunteers. Dig Dis Sci 1987; 32: 583-92.

16 Benjamin SB, Gerhardt DC, Castell DO. High amplitude peristaltic esophageal contractions associated with chest pain and/or dysphagia. Gastroenterology 1979; 77: 478-83.

17 Stuart RC, Hennessy TPJ. Primary disorders of oesophageal motility. Br f Surg 1989; 76: 1111-20.

18 Peters L, Maas L, Petty D, Dalton C, Penner D, Wu W, et al. Spontaneous noncardiac chest pain. Evaluation by 24 hour ambulatory esophageal motility and $\mathrm{pH}$ monitoring. Gastroenterology 1988; 94: 878-86.

19 DeMeester TR, O'Sullivan GC, Bermudez G, Midell AI, Cimochowski GE, O'Drobinak J. Esophageal function in patients with angina-type chest pain and normal coronary patients with angina-type chest pain and nor
angiogram. Ann Surg 1982; 196: 488-98.

20 Smout AJ, Breediik M, Van Der Zouw C. Physiologic gastro-esophageal reflux and esophageal motor activity studied with a new system for 24 -hour recording and automated analysis. Dig Dis Sci 1989; 34: 372-8. 